Ann. Biol. anim. Bioch. Biophys., I970, 10 (3), 385-399.

\title{
EFFETS DU MAINTIEN A POIDS CONSTANT PENDANT DE LONGUES DURÉES CHEZ LE RAT SEVRÉ. REPRISE DE LA CROISSANCE
}

\author{
G. DURAND et Êliane PENOT \\ avec la collaboration technique de Noëlle Bourgeaux \\ Station centrale de Nutrition, \\ Centre national de Recherches zootechniques, 78 -Jouy-en-Josas \\ Institut national de la Recherche agronomique
}

RÉSUMÉ

Des lots de rats mâles Wistar sevrés depuis une semaine, pesant $68 \mathrm{~g} \pm 2 \mathrm{~g}$, sont maintenus à poids constant uniquement par réduction des éléments énergétiques de la ration (lipides et glucides) pendant 50, 100 ou I60 jours. A la fin de chacune de ces périodes un lot est abattu et un lot est réalimenté ; les trois lots réalimentés sont abattus à $600 \mathrm{~g}$.

Le corps des animaux est divisé en six compartiments : peau, viscères + sang, foie, musculature des membres postérieurs, carcasse (musculature + squelette), tissus adipeux.

Ces compartiments sont comparés aux compartiments correspondants d'animaux témoins de même poids. Les variations du poids frais, ainsi que les quantités d'ADN, de protéines et d'ARN de chacun de ces compartiments sont mesurées. Ces mesures permettent en particulier d'évaluer les variations du nombre et de la taille des cellules.

Les principaux résultats indiquent :

I. Chez les animaux maintenus à $68 \mathrm{~g}$ :

a) La disparition apparente des tissus adipeux dès le $5^{\circ}$ jour.

b) Dans les cinq autres compartiments : un arrêt général de l'hyperplasie, le nombre des cellules restant constant dans les compartiments viscères + sang, foie et musculature, mais diminuant dans la peau ( -28 p. Ioo) et la carcasse (de - ro à-20 p. roo); une augmentation du contenu en protéines ( $+50 \mathrm{p}$. Ioo dans la musculature) ; une diminution du contenu en ARN (de - Io p. Ioo à - 57 p. Ioo selon les compartiments).

2. Chez les animaux réalimentés :

a) Une vitesse de croissance normale, lorsque la durée du maintien à poids constant qui précède la réalimentation, est inférieure à roo jours.

b) Une stimulation de la croissance des tissus adipeux et une diminution de la croissance musculaire.

c) Une forte reprise de l'hyperplasie (augmentation du nombre de cellules), même chez les animaux auparavant maintenus à $68 \mathrm{~g}$ pendant 160 jours; la capacité d'hyperplasie est conservée intégralement, dans la plupart des compartiments; elle est cependant nettement amoindrie dans la musculature. 


\section{INTRODUCTION}

Dans une première partie d'un précédent travail (DuRAND et al., I969) nous avions étudié le corps éviscéré, le foie et les muscles de Rats mâles maintenus, peu après sevrage, au poids de $70 \mathrm{~g}$ pendant 42 jours. Ces animaux étaient maintenus à poids constant uniquement par réduction de l'apport énergétique de la ration. Les principaux résultats montraient un arrêt de l'hyperplasie dans le foie et les muscles, et, dans le corps éviscéré, non seulement un arrêt global de l'hyperplasie, mais encore une diminution de $5 \mathrm{p}$. Ioo de la quantité globale de cellules. Dès lors, la question se posait de savoir quels tissus régressaient sous l'action de la carence énergétique.

Dans une deuxième partie, des animaux préalablement maintenus au poids de $70 \mathrm{~g}$ pendant 42 jours étaient réalimentés ad libitum et sacrifiés 286 jours plus tard, lorsqu'ils atteignaient le poids vif moyen de $700 \mathrm{~g}$. Il apparaissait alors que $I^{0}$ la vitesse de croissance moyenne de ces animaux, calculée sur l'ensemble de la période, n'était pas sensiblement différente de celle de témoins abattus au même poids ; $2^{0}$ la répartition des tissus n'était pas sensiblement modifiée par rapport aux témoins, cependant que la quantité de cellules était légèrement moindre dans les muscles (- II p. roo).

Le présent travail est destiné à préciser les données du précédent. Il concerne:

I. l'évolution des tissus et de leurs cellules chez des Rats mâles maintenus à poids constant après le sevrage ( 4 semaines, $68 \mathrm{~g}$ ), pendant des durées allant de $5 \mathrm{p}$. Ioo à 16 p. Ioo de l'espérance moyenne de vie de l'espèce ;

2. 1'influence de la durée de maintien à poids constant sur la capacité ultérieure de développement du Rat entier et de ses divers tissus.

Nous avons mesuré pour chaque compartiment étudié : - le poids frais, - le contenu protéique $(\mathrm{N} \times 6,25)-$ le contenu en $A D N$, proportionnel au nombre de cellules (BoIvin et al., I948), - le contenu en ARN.

Les variations des rapports poids frais/ADN, protéines/ADN, ARN/ADN permettent d'apprécier respectivement les variations de la taille des cellules, de leur contenu en protéines et en ARN.

\section{MATÉRIEI, ET MÉTHODES}

Matériel animal (tabl. I. - fig. I.)

Huit lots de rats mâles Wistar sont constitués à l'aide d'animaux âgés de 28 jours, sevrés depuis une semaine et pesant $68 \mathrm{~g} \pm 2 \mathrm{~g}$. Un lot (I A) est sacrifié le jour même. Un deuxième lot (II A) reçoit ad libitum un régime semi-synthétique équilibré contenant $\mathrm{r} 3 \mathrm{p}$. roo de matières azotées totales ; ce régime constitue le régime témoin (tabl. I). Les six autres lots sont maintenus au poids constant de $68 \mathrm{~g}$ uniquement par réduction des éléments énergétiques de la ration (lipides et glucides) pendant 50 , roo ou 160 jours. 
TABLEAU I

Composition du régime témoin en g/kg de matière sèche

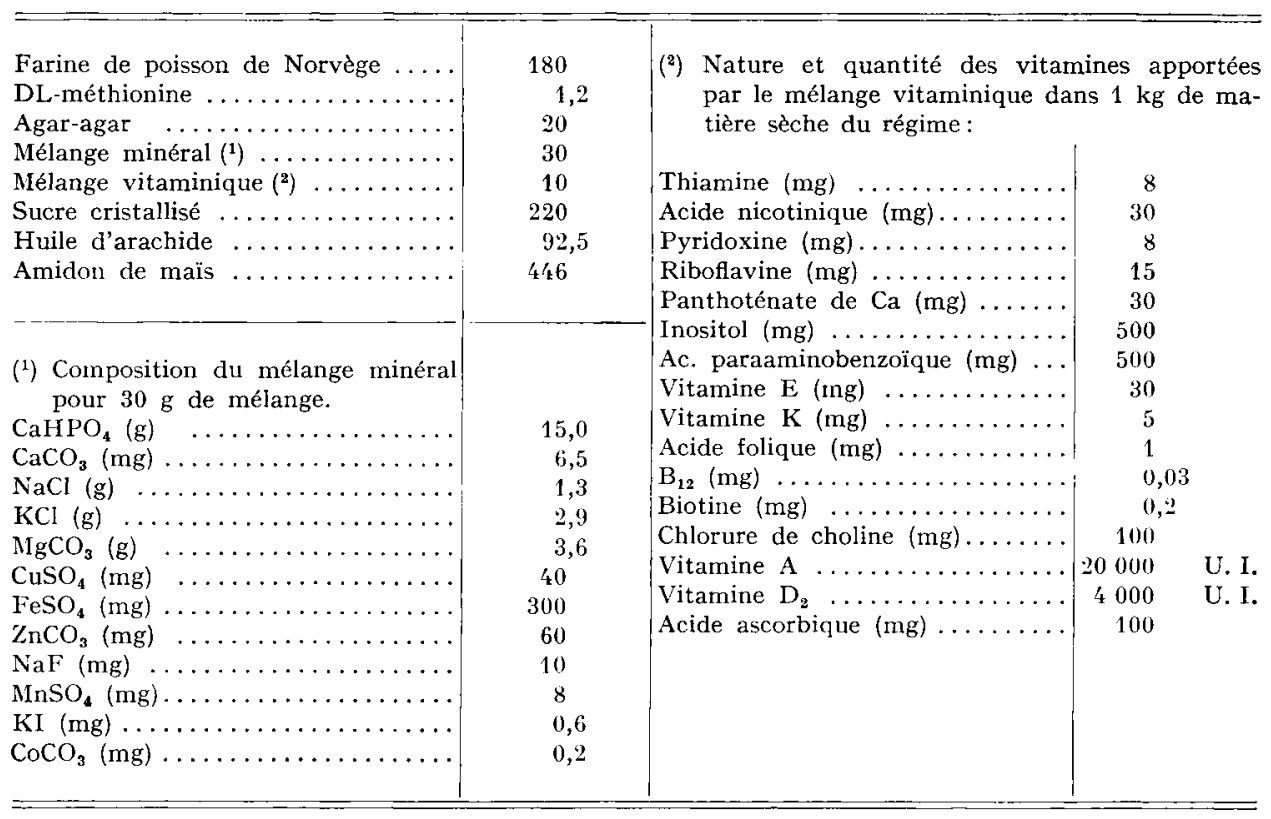

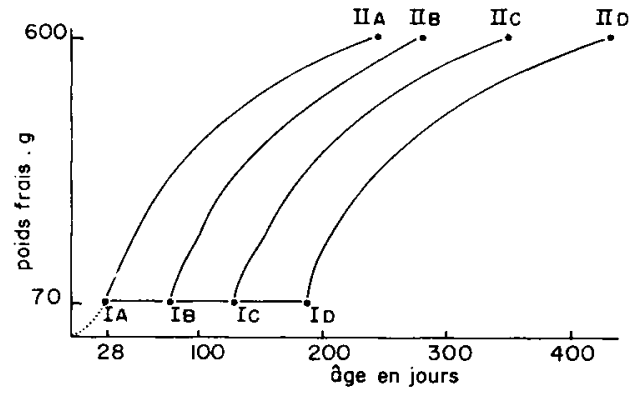

FIG. I. - Courbes de croissance

A la fin de chacune de ces périodes, un lot est sacrifié ( I B, I C, I D) et un autre réalimenté (Ir B, II C, II D). Le lot témoin II A et les lots réalimentés sont sacrifiés lorsque leur poids moyen atteint $600 \mathrm{~g}$; seuls sont retenus pour les analyses les animaux dont le poids s'écarte de moins de ro p. Ioo de la moyenne.

Les animaux sont élevés dans des cages en grillage métallique.

\section{Distribution des aliments}

Les rats normaux de $68 \mathrm{~g}$ consomment ro $\mathrm{g}$ du régime témoin. Pour maintenir les rats au poids constant de $68 \mathrm{~g}$ uniquement par réduction des éléments énergétiques de la ration, on leur distribue chaque jour la même quantité de protéines, minéraux et vitamines que consommeraient des animaux témoins de même poids. La quantité d'éléments énergétiques (amidon de maïs, saccharose et huile d'arachide) est ajustée chaque jour de façon que le poids des animaux pesés à jeun chaque matin reste constant. La proportion entre les trois sources énergétiques est toujours respectée. En pratique il faut distribuer i 5 à $20 \mathrm{p}$. roo des calories qu'ingèrent les témoins nourris ad libitum. Dans ces conditions, le taux protéique du régime restreint est de l'ordre de 35 p. Ioo. 


\section{Abattage et prélèvements}

Chaque animal, anesthésié à l'éther, est saigné. Le sang est recueilli dans de l'azote liquide. Puis le corps est réparti en six fractions distinctes :

- la peau;

- les dépôts adipeux, comprenant : les dépôts sous-cutanés périrénaux, épididymaires et mésentériques ;

- le foie ;

- les viscères, que l'on adjoint au sang ;

- la musculature des membres postérieurs;

- le reste du corps, appelé "carcasse " et qui comprend principalement le squelette et la musculature (noins la musculature des membres postérieurs).

Chaque partie est pesée et plongée dans l'azote liquide. Pour chaque lot, les six compartiments sont rassemblés dans autant de vases Dewar, avant traitements et analyses. Lorsque les traitements ne peuvent être effectués sur-le-champ, les échantillons sont conservés à $-{ }^{\circ} 8^{\circ} \mathrm{C}$ pendant une durée qui n'excède pas une semaine.

Les méthodes analytiques ont déjà été décrites (Durand et al., 1969).

\section{RÉSULTATS}

\section{I. - Effets du maintien à poids constant}

\section{Aspect des animaux.}

La longueur du corps (du nez à l'anus) n'augmente pas sensiblement. On note seulement au cours des premières semaines de poids constant, un allongement de la queue (tabl. 2), des oreilles et des poils. Mais dans l'ensemble, les animaux conservent un aspect assez voisin de celui des témoins de même poids. Leur activité physique est plutôt supérieure à celle des témoins, le taux de mortalité est nul.

Ainsi, les Rats s'adaptent bien au traitement qui leur est imposé.

TABLEAU 2

Maintien à poids constant

Numérotage et caractéristiques des lots. I A : lot témoin - I B, I C, I D : lots maintenus à poids constant $(68 \mathrm{~g})$ après le sevrage pendant respectivement 50 , Ioo ou 160 jours

\begin{tabular}{|c|c|c|c|c|}
\hline & I A & I $\mathrm{B}$ & I C & I $\mathrm{D}$ \\
\hline $\begin{array}{l}\text { Durée du maintien à poids } \\
\text { constant }(\mathrm{j}) \ldots \ldots \ldots \ldots\end{array}$ & 0 & 50 & 100 & 160 \\
\hline Poids vif $(\mathrm{g}) \ldots \ldots \ldots \ldots$ & 68,4 & 69,5 & 68,0 & 69,2 \\
\hline PVR (1) (g) $\ldots \ldots \ldots$ & 66,0 & 66,5 & 65,3 & 66,5 \\
\hline PVR p. 100 poids vif. & 96,5 & 95,7 & 96,0 & 96,1 \\
\hline Longueur totale $(\mathrm{cm}) \ldots$ & 23,5 & 27,3 & 27,4 & 27,6 \\
\hline Longueur $\mathrm{du}$ corps $(\mathrm{cm})$ & 43,8 & 14,6 & 14,0 & 14,9 \\
\hline Age $(j) \ldots \ldots \ldots \ldots \ldots$ & 28 & $28+50$ & $28+100$ & $28+160$ \\
\hline Nombre d'animaux ..... & 10 & 10 & 10 & 10 \\
\hline
\end{tabular}

( $\left.{ }^{1}\right)$ PVR $=$ poids vif reconstitué (obtenu en additionnant les poids frais des six compartiments corporels constitués après dissection). 
2. Organes génitaux (fig. 2).

Les testicules, qui font partie du compartiment " viscères + sang", sont pesés séparément. L'évolution de leur poids est portée sur la figure 2 . Il apparaît que les gonades continuent de croître au cours des premiers jours de maintien à poids constant, atteignant 2,5 fois le poids des testicules des témoins au $5^{\circ}$ jour. Au-delà, on enregistre une décroissance pondérale très vive qui, après I6o jours, ramène les testicules à une taille infantile.

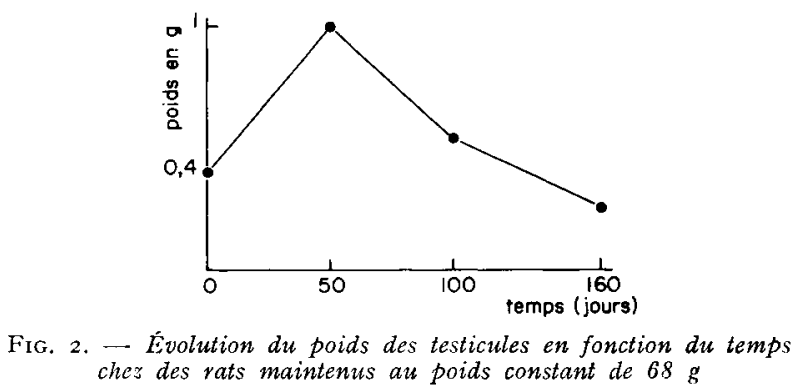

Après Io semaines environ de maintien à poids constant, le pénis devient saillant. Ce phénomène a déjà été observé et étudié chez des Rats soumis à une sousalimentation générale (WIDDowson et al., 1964).

3. Action sur les compartiments corporels (fig. 3).

a) Peau (fig. 3 a).

Le poids frais de ce compartiment diminue progressivement, l'écart avec les témoins atteignant environ $20 \mathrm{p}$. Ioo après I 60 jours. I e contenu protéique dépasse de $44 \mathrm{p}$. Ioo celui des témoins après 50 jours de maintien à poids constant. Cette nette supériorité se conserve jusqu'au $\mathrm{IOO}^{\mathrm{e}}$ jour, mais s'atténue par la suite, l'écart n'étant plus que de $20 \mathrm{p}$. Ioo après I6o jours.

Les quantités d'acides nucléiques sont amoindries. La quantité d'ADN décroît progressivement entre le $50^{\circ}$ et le roo jour ; la perte atteint $28 \mathrm{p}$. roo au $\mathrm{IOo}^{\mathrm{e}}$ jour, mais ne se poursuit pas au-delà. L'ARN chute de 57 p. roo au cours des 50 premiers jours, mais n'évolue pratiquement pas par la suite.

b) Viscères + sang (fig. 3 b).

On note pour ce compartiment, au cours des 50 premiers jours, des accroissements de I2 p. roo environ du poids frais et de $23 \mathrm{p}$. Ioo du contenu protéique. Après le $5^{\mathrm{e}}$ jour, il n'y a pas d'évolution notable de ces deux caractéristiques.

Les quantités d'acides nucléiques ne diffèrent pas, au $50^{\mathrm{e}}$ jour, de ce qu'elles sont chez les témoins. Par la suite, on relève une légère diminution, de l'ordre de Io p. I00.

c) Foie (fig. $3 \mathrm{c}$ ).

Le poids frais de l'organe décroît de 20 p. Ioo au cours des 50 premiers jours ; cependant, l'écart avec les témoins s'atténue ensuite progressivement et ne dépasse pas Io p. I00 au I $60^{\mathrm{e}}$ jour. Le contenu du foie en protéines augmente de 5 à Io p. Ioo 

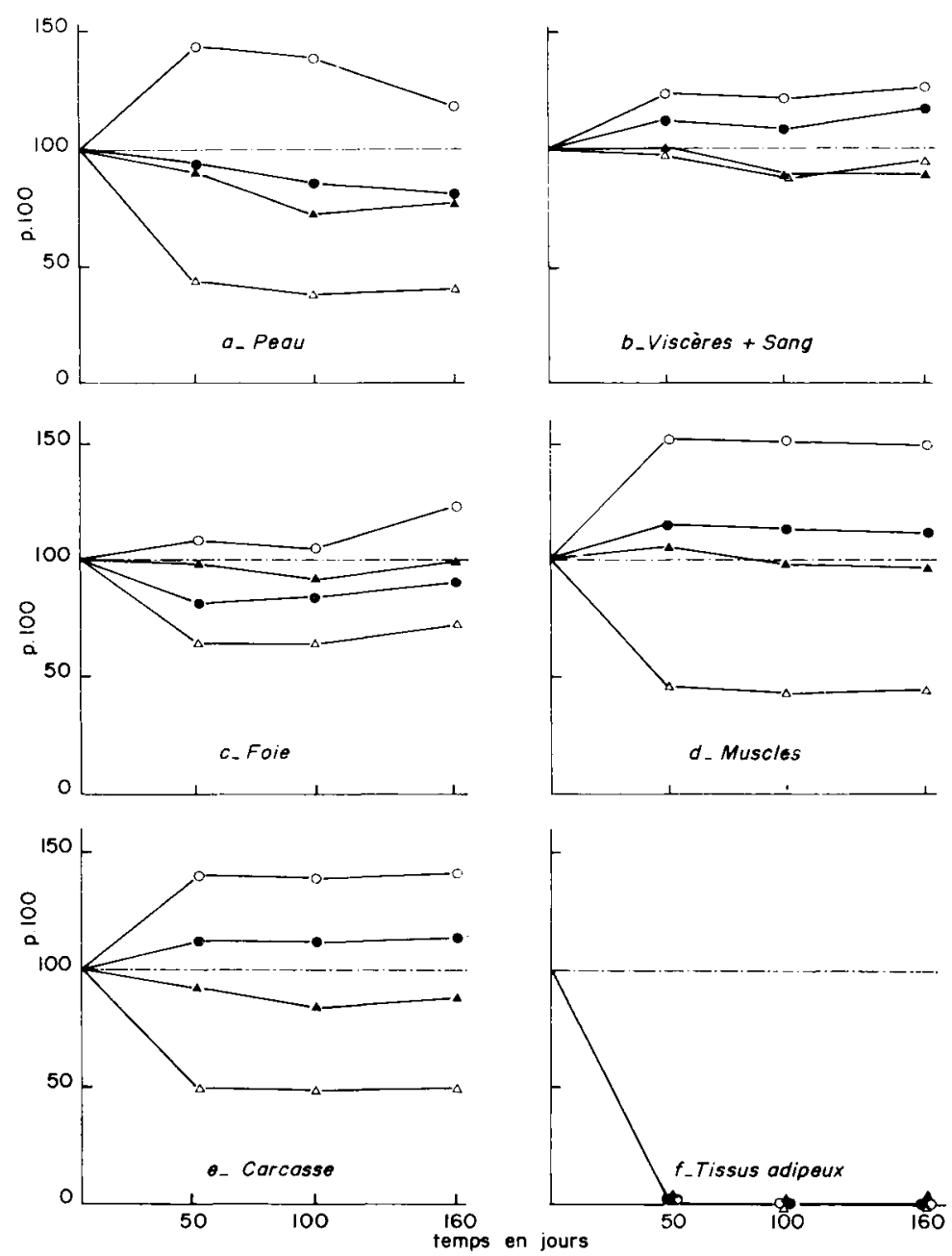

Fig. 3. - Maintien à poids constant: évolution en fonction du temps, du poids frais ( $\bullet)$, des contenus en protéines (O), en $A D N(\Delta)$, et en $A R N(\Delta)$ de divers compartiments corporels chez le rat mâle maintenu au poids constant de $68 \mathrm{~g}$. Les données sont exprimées en pourcentage de jeunes animaux témoins de même poids.

au cours des Ioo premiers jours de maintien à poids constant, mais subit entre le ${ }^{100}{ }^{\mathrm{e}}$ et $1 \mathrm{e} \mathrm{I} 6 \mathrm{o}^{\mathrm{e}}$ jour un accroissement important qui le situe à $23 \mathrm{p}$. Ioo au-dessus des témoins. En ce qui concerne les acides nucléiques, on constate tout d'abord que le contenu en $A D N$ n'est pas modifié ; la quantité d'ARN, par contre, décroît de 35 p. roo environ au cours des 50 premiers jours, se maintient pratiquement à ce niveau jusqu'au ${ }{ }^{\mathrm{e}} \mathrm{e}$ jour, puis tend à augmenter, comme le contenu protéique, entre le roo et le ${ }^{\mathrm{e}} 6 \mathrm{o}^{\mathrm{e}}$ jour.

d) Muscles (fig. $3 d$ ).

Le poids frais de la musculature, tout au moins celle des membres postérieurs, est augmenté de $I 5 \mathrm{p}$. Ioo à la fin des $5^{\circ}$ premiers jours de maintien à poids constant. Cette supériorité tend à diminuer très progressivement au cours des mois suivants, 
puisqu'elle n'est plus que de II p. Ioo après I6o jours. Le contenu protéique musculaire s'accroît de 50 p. Ioo durant les 50 premiers jours et se stabilise ensuite. La quantité d'ADN demeure remarquablement stable pendant toute la période étudiée ; la quantité d'ARN est amoindrie de $55 \mathrm{p}$. Ioo environ au $5^{0^{\mathrm{e}}}$ jour et n'évolue pratiquement pas par la suite.

e) Carcasse (fig. $3 e$ ).

Ce compartiment réagit au maintien à poids constant d'une façon comparable à celle du compartiment précédent en ce qui concerne poids frais, contenu protéique et ARN. Cependant les écarts sont moins accusés pour l'augmentation du contenu protéique $(+40 \mathrm{p}$. Ioo au lieu de $+50 \mathrm{p}$. Ioo) et la diminution de l'ARN $(-50 \mathrm{p}$. Ioo au lieu de $-55 \mathrm{p}$. IOo). Ceci incline à penser que le squelette joue un rôle modérateur quant à ces deux points. Contrairement au compartiment "muscles ", la carcasse perd une quantité appréciable d'ARN - (entre ro et 20) - qui, suivant toute vraisemblance, provient du squelette.

f) Tissus adipeux (fig. $3 f$ ).

Après le $5^{\mathrm{e}}$ jour de maintien à poids constant, les tissus adipeux ont apparemment disparu.

\section{II. - Effets de la réalimentation ad libitum}

I. Vitesse de croissance (fig. I, tab1. 3).

Il faut 2 I 6 jours aux lots témoins (II A) pour croître de $68 \mathrm{~g}$ à $600 \mathrm{~g}$. Les lots II $\mathrm{B}$ et II $\mathrm{C}$ mettent une durée sensiblement équivalente, le lot II B croissant même légèrement plus vite. Par contre, la croissance du lot II D est plus lente que celle du lot témoin (- II p. Ioo). On remarque, par ailleurs, que les courbes de croissance

\section{TABLEAU 3}

Récupération

Numérotage et caractéristiques des lots II $\mathrm{A}:$ lot témoin - il $\mathrm{B}$, II $\mathrm{C}$, II $\mathrm{D}$ : lots réalimentés après avoir été maintenus à poids constant après le sevrage (68 j) pendant respectivement 50 , I00 ou 160 jours

\begin{tabular}{|c|c|c|c|c|}
\hline & II A & II $\mathrm{B}$ & II C & II D \\
\hline Poids vif $(g)$ & 596 & 597 & boo & 595 \\
\hline PVR $\left({ }^{1}\right)(g) \ldots \ldots \ldots \ldots$ & 576 & 577 & 584 & 579 \\
\hline PVR p. 100 poids vif .. & 96,6 & 96,6 & 97,3 & 97,3 \\
\hline Longrueur totale $(\mathrm{cm}) \ldots$ & 46 & 45 & 43 & 42 \\
\hline Longueur du corps $(\mathrm{cm})$. & 25,9 & 25,1 & 25,4 & 25,2 \\
\hline Age $(j) \ldots \ldots \ldots \ldots \ldots$ & $2 / 14$ & 280 & 348 & 428 \\
\hline Durée $68 \mathrm{~g}-600 \mathrm{~g}(2)$ (j) ... & 216 & 202 & 220 & 240 \\
\hline Nombre d'animaux ..... & 6 & 6 & 7 & 5 \\
\hline
\end{tabular}

(1) $\mathrm{PVR}=$ poids vif reconstitué (obtenu en additionnant le poids frais des six compartiments corporels constitués après dissection.

${ }^{2}$ ) Temps nécessaire aux animaux pour passer de $685 \mathrm{~g}$ à $600 \mathrm{~g}$. 
présentent une forme particulière propre aux animaux en croissance compensatrice qui ont été préalablement maintenus à un poids ou la maturité sexuelle ne peut être atteinte (DURAND et al., I969).

\section{Aspect des animaux (tabl. 3).}

La longueur totale des animaux des lots II A et II B est équivalente. Cependant les animaux des lots II C et II D sont plus courts. Mais il apparaît que la différence de longueur entre les uns et les autres n'est due qu'à la queue ; la longueur du corps, du nez à l'anus, étant la même chez les quatre lots.

Hormis ce point, on ne note pas de différence apparente dans l'aspect extérieur des animaux, quel qu'ait été leur passé nutritionnel.

\section{Organes génitaux.}

La réalimentation fait disparaître complètement les effets visibles du maintien à poids constant sur le pénis. Cependant, alors que le poids moyen des testicules est de $3,2 \mathrm{~g}$ pour les témoins, il n'est que de $2,7 \mathrm{~g}$ pour les rats du lot II $\mathrm{B}$; le poids des gonades des lots II C et II D n'a pas été mesuré.

4. Effets sur les compartiments corporels (fig. 4, tabl. 4).

a) Peau (fig. 4 a).

Quel que soit le stade auquel la réalimentation a eu lieu, les quatre principales caractéristiques étudiées (poids frais, contenus en protéines, en ADN et en ARN) sont comparables chez les animaux expérimentaux et chez les témoins. Ainsi, la peau, dont nous avons vu plus haut qu'elle était un des tissus les plus sensibles à la sous-nutrition énergétique, récupère parfaitement.

b) Viscères + sang (fig. 4 b).

Les valeurs des poids frais et des contenus en protéines sont assez dispersées chez les trois lots réalimentés. Les quantités d'ADN et surtout d'ARN sont inférieures à celles des témoins. Pour l'ARN, l'écart est d'autant plus grand que les animaux sont maintenus plus longtemps à poids constant : la valeur maximale de cet écart atteint $20 \mathrm{p}$. Ioo.

\section{c) Foie (fig. 4 c).}

La malnutrition énergétique de longue durée, après le sevrage, semble favoriser la croissance ultérieure du foie. Ainsi, les organes des animaux expérimentaux sont plus lourds et plus riches en protéines (de ro à 20 p. Ioo), lorsque la durée de maintien à poids constant atteint roo jours ; de même ils ont tendance à contenir plus d'ADN (de 5 à Io p. IOo). L'ARN ne suit pas cette tendance et se contente de revenir à la normale.

d) Muscles (fig. $4 d$ ).

Contrairement à celle du foie, la croissance musculaire semble irrémédiablement défavorisée lorsque la consommation d'aliments énergétiques est limitée pendant une longue période après le sevrage. L,es effets se font déjà faiblement sentir sur les quatre caractéristiques étudiées et en particulier sur l'ADN lorsque le maintien à $68 \mathrm{~g}$ est limité à 50 jours. Les écarts sont franchement plus accusés lorsque le maintien à poids constant atteint roo jours : la masse de la musculature des 


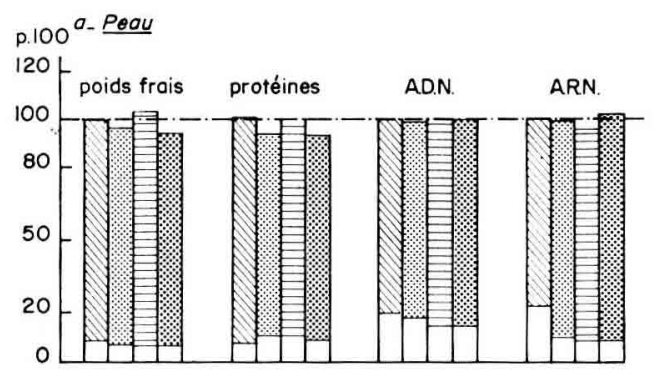

b. Viscöres + Sang
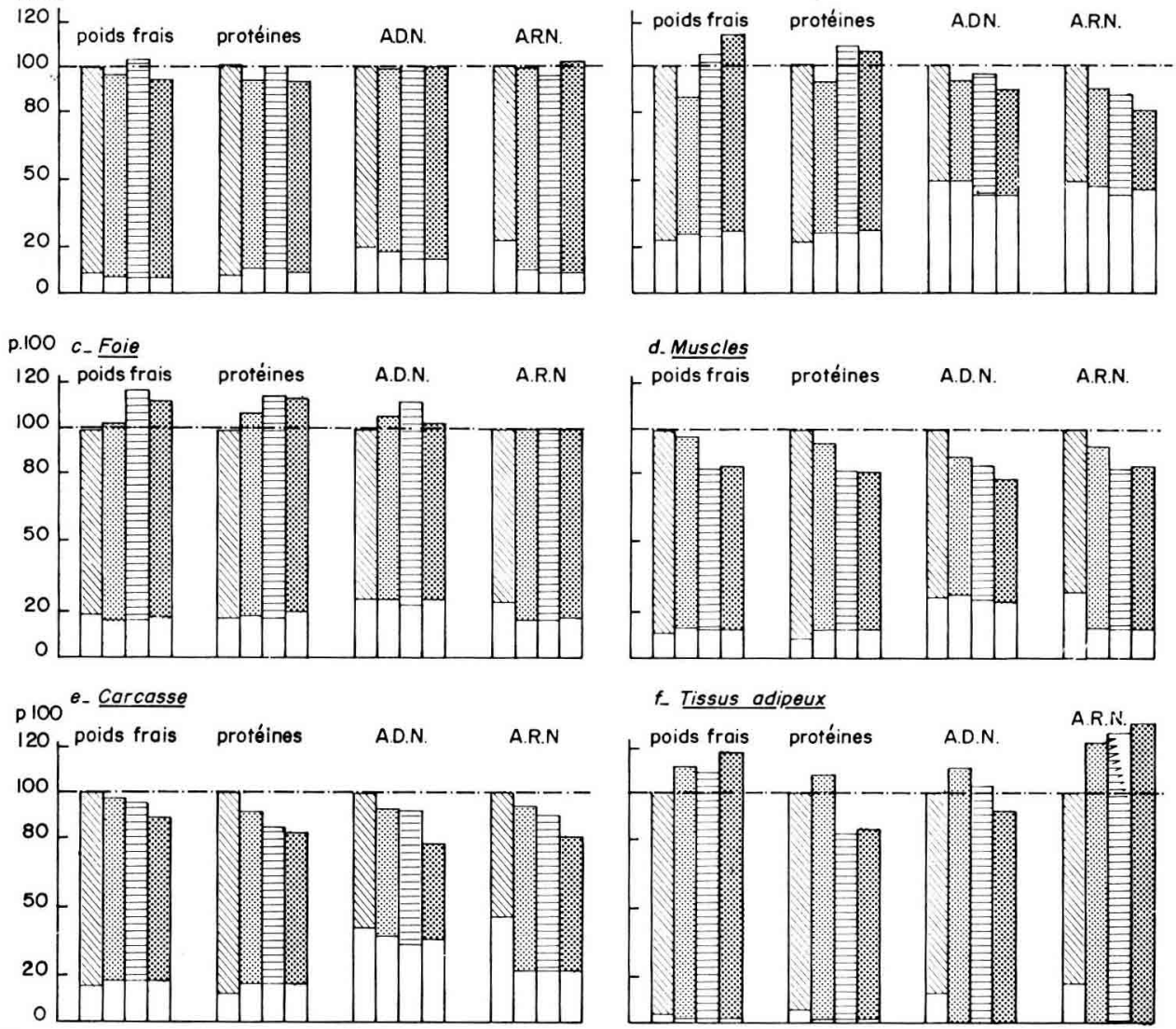

FIG. 4. - Récupération: poids frais et contenus en protéines $A D N$ et $A R N$ chez le rat adulte $(600 \mathrm{~g})$ normal ou préalablement maintenu à poids constant $(68 \mathrm{~g})$ après le sevrage pendant $50($ ), 100 ), et 160 jours ( 1 Les données sont exprimées en pourcentage de témoins de même poids ( La partie inferieure des colonnes ( données chez les animaux de 68 g correspondants.

) représente la valeur des

membres postérieurs, les contenus en protéines, en ADN et en ARN sont alors amoindris de $I 5$ à 20 p. Ioo. Il y a peu de différences entre les stades Ioo et I6o jours, sauf pour l'ADN, d'autant moins abondant que la période de restriction a été plus longue. L'écart qui sépare le lot II D du lot témoin II A atteint 22 p. roo.

e) Carcasse (fig. $4 e$ ).

Ce compartiment est composé pour l'essentiel de la musculature (hormis celle des membres postérieurs) et du squelette. Dans les grandes lignes, ses réactions, face aux conditions nutritionnelles imposées, sont comparables à celles des muscles. Cependant, le squelette paraît jouer un rôle modérateur, et l'évolution des divers points étudiés est progressive ; ceci semble indiquer que le squelette récupère 
mieux que la musculature, à condition que la durée de sous-nutrition imposée au sevrage n'excède pas roo jours.

\section{f) Tissus adipeux (fig. $4 f$ ).}

Le poids frais des tissus adipeux est de Io à 20 p. Ioo supérieur chez les animaux réalimentés par rapport à ce qu'il est chez les témoins, mais entre 50 et

TABI,EAU 4

Maintien à poids constant

Teneurs en acides nucléiques et en protéines dans les tissus du rat maintenu à poids constant (68 g) après le sevrage :

I $\mathrm{A}=$ lot témoin $-\mathrm{I} \mathrm{B}, \mathrm{I} \mathrm{C}, \mathrm{I} \mathrm{D}=$ lots maintenus à poids constant respectivement pendant 50 , Ioo ou 160 jours

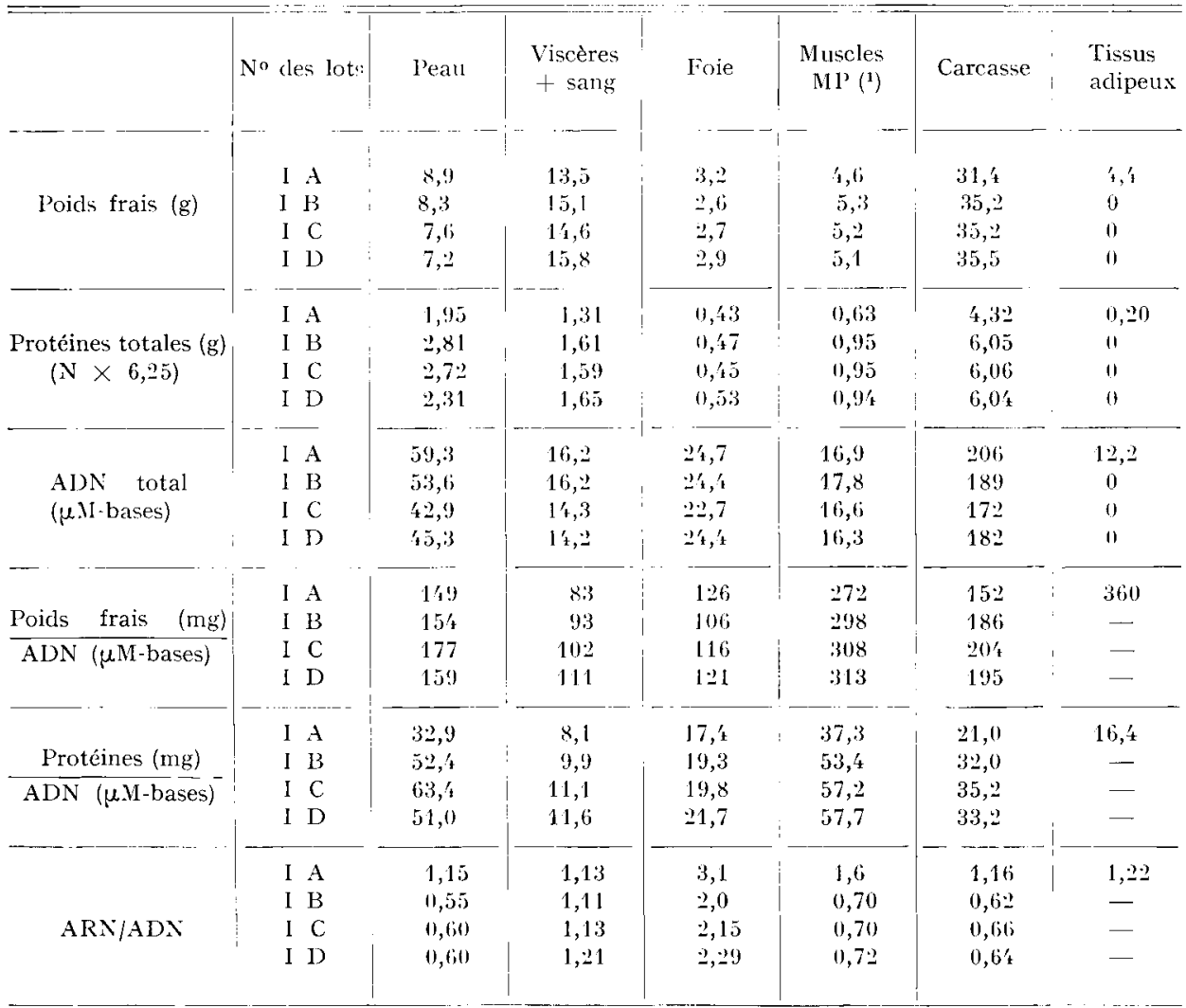

(1) $\mathrm{MP}=$ Membres postérieurs.

I6o jours, la durée de maintien à poids constant ne semble pas avoir une importance particulière. En ce qui concerne le contenu protéique, on remarque que la sousalimentation énergétique préalable de 50 jours a un effet bénéfique $(+8 \mathrm{p}$. IOo), tandis que sur une plus longue durée, l'effet devient défavorable (- I5 à 20 p. IOo). 
Les tissus adipeux des animaux expérimentaux contiennent à peu près autant d'ADN que ceux des témoins, cependant que leur contenu en ARN est très nettement plus élevé (de 20 à 30 p. IOO).

\section{DISCUSSION}

\section{I. - Maintien à poids constant}

Dans le présent travail, des Rats mâles sont maintenus à poids constant ( $68 \mathrm{~g}$ ) une semaine après le sevrage pendant 50 , Ioo et 160 jours uniquement par réduction de l'apport énergétique de la ration. Ces durées de maintien à poids constant correspondent respectivement à environ 5 , Io et I6 p. Ioo de l'espérance de vie considérée comme normale pour les animaux utilisés (KAYSER et al., r966).

On constate qu'au cours de la période de restriction, et plus particulièrement entre le $\mathrm{I}^{\mathrm{er}}$ et le $5^{\mathrm{e}}$ jour, le poids frais de certains compartiments corporels (viscères + sang, musculature) continue de s'accroître tandis que, évidemment, d'autres régressent (peau, foie) ou disparaissent (tissus adipeux). Ces faits n'apportent qu'un argument supplémentaire à la notion de tissus prioritaires maintes fois démontrée depuis les travaux de MCMEEKAN (I940-4I). Il convient cependant de relever le rôle important que joue la peau dans cette redistribution tissulaire. Par ailleurs, il est bien évident que les résultats exposés ci-dessus à propos du compartiment viscères + sang, ensemble très hétérogène, ne constituent que des moyennes globales relatives à des organes dont les comportements respectifs peuvent être différents, voire opposés, face aux conditions nutritionnelles imposées.

De plus, le comportement d'un même organe peut varier au cours de la période de sous-nutrition énergétique. Ce fait est très bien illustré par l'évolution des testicules, dont le poids augmente au cours des 50 premiers jours et régresse ensuite. Notons à ce propos que l'opinion suivant laquelle une sous-nutrition sévère chez le Rat en croissance fait que les testicules représentent un pourcentage inhabituellement élevé du poids du corps (Siperstern, I92i ; Widdowson et al., I963), n'est pas générale; elle s'avère seulement lorsque la restriction est peu sévère ou de courte durée.

La restriction énergétique, telle que nous l'avons imposée aux animaux, provoque l'augmentation non seulement des teneurs, mais aussi des contenus protéiques dans cinq des six compartiments étudiés. Cette augmentation est particulièrement importante pour la peau ( +40 p. Ioo), les muscles des membres postérieurs $\left(+50 \mathrm{p}\right.$. IOO), et la carcasse $\left(+40 \mathrm{p}\right.$. Ioo dès le $50^{\mathrm{e}}$ jour $)$, soit en moyenne légèrement supérieure à $40 \mathrm{p}$. Ioo pour ces trois compartiments. Ceci confirme, en les renforçant, les résultats exposés dans une précédente publication (DuRANo et al., I969), suivant lesquels, dans les mêmes conditions expérimentales le contenu protéique du corps éviscéré des Rats maintenus à $70 \mathrm{~g}$ pendant 42 jours était augmenté de 23 p. Ioo.

On constate, par ailleurs, que dans aucun des compartiments, il n'y a augmentation de la quantité d'ADN, donc de cellules. L'arrêt de 1'hyperplasie est donc 
général. Mais tandis que la quantité de cellules reste pratiquement constante pendant la période de restriction, quelle que soit sa durée, dans trois compartiments (viscères + sang, foie, muscles), elle diminue dans les trois autres (peau, carcasse, tissus adipet1x) ; apparemment réduite à néant dans les tissus adipeux dès le $50^{\circ}$ jour, elle est amoindrie de 20 à $30 \mathrm{p}$. Ioo dans la peau et de Io à $20 \mathrm{p}$. Ioo dans la carcasse au Ioo $^{\mathrm{e}}$ jour. La carcasse étant constituée essentiellement de la musculature et du squelette, et I'ADN musculaire manifestant une parfaite résistance à la malnutrition, on peut penser que la perte de cellules dans ce compartiment provient du squelette.

La quantité d'ARN diminue de 50 à $60 \mathrm{p}$. Ioo dans les tissus, cependant qu'elle est préservée dans le compartiment viscères + sang. Nous avons déjà remarqué l'insensibilité globale de ce compartiment dans le cas d'une restriction énergétique très sévère appliquée à des Rates adultes (DuRAND et PENOT, I969).

Finalement, en faisant abstraction des tissus adipeux devenus inexistants, il ressort des résultats que :

$I^{0}$ la taille moyenne des cellules augmente dans quatre compartiments, mais diminue dans le foie ; $2^{0}$ le contenu et la teneur en protéines augmentent dans les cellules de cinq compartiments ; $3^{\circ}$ le contenu et la teneur en ARN diminuent dans quatre compartiments, mais restent pratiquement stables dans le compartiment viscères + sang.

\section{II. - Récupération}

Remarquons tout d'abord que la capacité d'hyperplasie est conservée totalement, ou en partie, dans tous les compartiments étudiés, chez des Rats âgés de I 88 jours, maintenus à $68 \mathrm{~g}$ pendant $\mathbf{I} 60$ jours. A cet âge, les animaux témoins pèsent $540 \mathrm{~g}$ environ, stade auquel l'hyperplasie est pratiquement arrivée à son terme dans la plupart des tissus (DuRAND et al., 1969).

D'autre part, il a déjà été démontré que, sous certaines conditions, des animaux dont le développement a été temporairement fortement ralenti ou arrêté par des carences nutritionnelles, ne peuvent atteindre ni la stature, ni le poids qui auraient été les leurs s'ils avaient été constamment bien alimentés (MCCAY et al., I953 ; Widdowson et McCANCE, I963; Lister et McCANCE, I967 ; Brown et Guthrie, Ig68; BARNes et al., I968).

Tous les auteurs s'accordent pour affirmer qu'à durée et sévérité relative égales, les effets ultérieurs d'une malnutrition passagère sont d'autant plus marqués que celle-ci a été imposée à un stade précoce du développement.

Dans les conditions expérimentales du présent travail qui comportent l'utilisation des Rats mâles sevrés et ayant disposé de quelques jours pour s'adapter à la vie indépendante, nous avons montré que les animaux peuvent être maintenus pendant au moins roo jours au poids de $68 \mathrm{~g}$ sans que leur poids définitif, après réalimentation, soit amoindri.

Mais que le poids définitif de ces animaux soit optimal ne signifie pas pour autant que la répartition pondérale de leurs organes et tissus soit semblable à celle de témoins normaux. Cela ne signifie pas non plus que toutes les caractéristiques de leurs tissus et organes soient elles aussi normales.

De l'examen de nos résultats, il apparaît en effet qu'après une sous-nutrition énergétique, suivie de réalimentation, la répartition pondérale finale des divers 
TABLEAU 5

Récupération

Teneurs en acides nucléiques et en protéines dans les tissus du rat adulte $(600 \mathrm{~g})$

I $\mathrm{A}=$ lot témoin - I B, I C, I D = lots préalablement maintenus au poids constant de $68 \mathrm{~g}$ pendant respectivement 50 , roo ou 160 jours.

\begin{tabular}{|c|c|c|c|c|c|c|c|}
\hline & $N^{\circ}$ des lots & Peau & $\begin{array}{l}\text { Viscères } \\
+ \text { sang }\end{array}$ & Foie & $\begin{array}{l}\text { Muscles } \\
\text { MP (1) }\end{array}$ & Carcasse & $\begin{array}{l}\text { Tissus } \\
\text { adipeux }\end{array}$ \\
\hline Poids frais $(\mathrm{g})$ & $\begin{array}{ll}\text { II } & \mathrm{A} \\
\text { II } & \mathrm{B} \\
\text { II } & \mathrm{C} \\
\text { II } & \mathrm{D}\end{array}$ & $\begin{array}{r}102 \\
98 \\
105 \\
96\end{array}$ & $\begin{array}{l}58,1 \\
50,0 \\
61,5 \\
66,1\end{array}$ & $\begin{array}{l}16,5 \\
17,0 \\
19,5 \\
18,7\end{array}$ & $\begin{array}{l}42,2 \\
40,9 \\
35,1 \\
35,5\end{array}$ & $\begin{array}{l}201 \\
197 \\
193 \\
179\end{array}$ & $\begin{array}{l}156 \\
17 \prime^{\prime} \\
170 \\
184\end{array}$ \\
\hline $\begin{array}{l}\text { Protéines totales }(\mathrm{g}) \\
\qquad(\mathrm{N} \times 6, \geq 5)\end{array}$ & $\begin{array}{ll}\text { II } & A \\
\text { II } & B \\
\text { II } & C \\
\text { II } & D\end{array}$ & $\begin{array}{l}24,5 \\
24,4 \\
2 !, 6 \\
23,9\end{array}$ & $\begin{array}{l}6,1 \\
5,7 \\
6,6 \\
6,5\end{array}$ & $\begin{array}{l}2,6 i \\
2,8 \\
3,0 \\
2,7\end{array}$ & $\begin{array}{l}7,9 \\
7,4 \\
6,5 \\
6,4\end{array}$ & $\begin{array}{l}35,2 \\
32,3 \\
30,0 \\
29,1\end{array}$ & $\begin{array}{l}3,8 \\
4,1 \\
3,1 \\
3,2\end{array}$ \\
\hline $\begin{array}{l}\text { ADN total } \\
\text { ( } \mu \mathrm{NI}-\text { bases) }\end{array}$ & $\begin{array}{ll}\text { II } & A \\
\text { II } & B \\
\text { II } & C \\
\text { II } & D\end{array}$ & $\begin{array}{l}291 \\
293 \\
289 \\
295\end{array}$ & $\begin{array}{l}33 ! \\
311 \\
32 \% \\
396\end{array}$ & $\begin{array}{r}98 \\
104 \\
110 \\
101\end{array}$ & $\begin{array}{l}67 \\
59 \\
56 \\
52\end{array}$ & $\begin{array}{l}505 \\
471 \\
465 \\
392\end{array}$ & $\begin{array}{r}106 \\
118 \\
109 \\
97\end{array}$ \\
\hline$\frac{\text { Poids }}{\text { ADN }} \frac{\text { frais }}{\mu M \text {-bases }}$ & $\begin{array}{ll}\text { II } & A \\
\text { II } & B \\
\text { II } & C \\
\text { II } & D\end{array}$ & $\begin{array}{l}347 \\
33 t \\
36 t^{\prime} \\
325\end{array}$ & $\begin{array}{l}175 \\
161 \\
191 \\
223\end{array}$ & $\begin{array}{l}168 \\
163 \\
177 \\
185\end{array}$ & $\begin{array}{l}629 \\
693 \\
627 \\
682\end{array}$ & $\begin{array}{l}397 \\
418 \\
416 \\
458\end{array}$ & 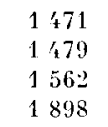 \\
\hline$\frac{\text { Protéines (mg) }}{\text { ADN ( } \text { ( M-bases) }}$ & $\begin{array}{ll}\text { II } & \mathrm{A} \\
\text { II } & \mathrm{B} \\
\text { II } & \mathrm{C} \\
\text { II } & \mathrm{D}\end{array}$ & $\begin{array}{l}83,3 \\
83,3 \\
85,1 \\
81,0\end{array}$ & $\begin{array}{l}18,3 \\
18,3 \\
20,4 \\
21,9\end{array}$ & $\begin{array}{l}26,5 \\
26,9 \\
27,2 \\
26,7\end{array}$ & $\begin{array}{l}118 \\
125 \\
121 \\
129\end{array}$ & $\begin{array}{l}69,7 \\
68,6 \\
64,5 \\
75,0\end{array}$ & $\begin{array}{l}35,8 \\
34,7 \\
28,1 \\
33,0\end{array}$ \\
\hline$A R N / A D N$ & $\begin{array}{ll}\text { II } & A \\
\text { I I } & B \\
\text { II } & C \\
\text { II } & \text { D }\end{array}$ & $\begin{array}{l}0,99 \\
0,98 \\
0,96 \\
1,00\end{array}$ & $\begin{array}{l}1,1^{\prime} t \\
1,09 \\
1,02 \\
1,02\end{array}$ & $\begin{array}{l}3,21 \\
3,02 \\
2,90 \\
3,07\end{array}$ & $\begin{array}{l}1,45 \\
1,53 \\
1,45 \\
1,59\end{array}$ & $\begin{array}{l}1,03 \\
1,0^{\prime} \mathrm{k} \\
1,02 \\
1,08\end{array}$ & $\begin{array}{l}0,87 \\
0,95 \\
1,06 \\
1,23\end{array}$ \\
\hline
\end{tabular}

(1) MP $=$ Membres postérieurs.

compartiments étudiés est généralement modifiée. Seule la peau redevient normale, quelle qu'ait été la durée de la restriction. Parmi les cinq autres compartiments, trois sont avantagés (viscères + sang, foie, tissus adipeux) et deux désavantagés (muscles des membres postérieurs et carcasse). Ce qui nous parait le plus important

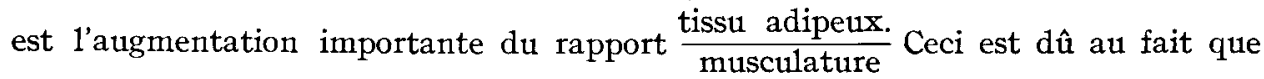
dans le muscle, la capacité d'hyperplasie est diminuée en raison inverse de la durée de la malnutrition, tandis que dans les tissus adipeux cette capacité est conservée.

Des recherches ont été entreprises pour déterminer comment évolue l'ensemble des phénomènes décrits ci-dessus lorsque les durées de maintien à poids constant s'étendent sur un et deux ans, ou plus si possible. 


\section{SUMMARY}

\section{EFFECT OF KEEPING WEANED RATS AT THE SAME WEIGHT FOR IONG PERIODS. RESUMPTION OF GROWTH}

Groups of male Wistar rats weighing $68 \pm 2 \mathrm{~g}$, which had been weaned for a week were maintained at constant weight by reducing the energy constituents of the diet (lipids and carbohydrates) during 50, I 50 or I 60 days. At the end of each of these periods one group was killed and one was refed; the three refed groups were killed at $600 \mathrm{~g}$.

The bodies of the animals were divided into six compartments : skin, viscera + blood, liver, muscles of the hind limbs, carcass (musculature and skeleton) and adipose tissue.

These compartments were compared with the corresponding compartments of control animals of the same weight. The variations in fresh weight, and also the amounts of deoxyribonucleic acid (DNA), protein and ribonucleic acid (RNA) of each of the compartments was measured. These measurements made it possible to evaluate the variations in the number and size of the cells.

The main results showed :

I. In the animals maintained at $68 \mathrm{~g}$ :

a) Apparent disappearance of adipose tissue after the 5 oth day.

b) In the other five compartments : - a general cessation of hyperplasia, the number of cells remaining constant in the compartments viscera + blood, liver and musculature, but decreasing in skin ( $-28 \mathrm{p} .100)$ and in carcass ( - Io to $-20 \mathrm{p}$. I0o). There was an increase in protein content $(+50 \mathrm{p}$. 100 in musculature) and a decrease in RNA content ( - Io to - 5 I p. roo in different compartments).

2. In the refed animals :

a) A normal rate of growth when weight before refeeding had been kept constant for less than Ioo days.

b) Stimulation of growth of adipose tissue and diminution of growth of muscle.

c) A marked resumption of hyperplasia (increase in number of cells) even in rats formerly maintained at $68 \mathrm{~g}$ for $\mathrm{I} 60$ days. The capacity for hyperplasia was not impaired in most compartments; it was, however, reduced in musculature.

\section{RÉFÉRENCESS BIBLIOGRAPHIQUES}

Barnes H. R., Neely C. S., Kwong E., Labadan B. A., Slavka F., Ig68. Postnatal nutritional deprivations as determinants of adult rat behavior toward food, its consumption and utilization. $J$. Nutr., 96, 467-476.

Borvin A., VEndRely R., VendRely C., I948. I'acide désoxyribonucléique du noyau cellulaire dépositaire des caractères héréditaires; arguments d'ordre analytique. C. R. Acad. Sci. Fr., 226, Io6II06:

Brown M. L., Guthrie H. A., I968. Effect of severe undernutrition in early life upon body and organ weights in adult rats. Growth, 32, I43-I50.

Durand G., Fauconneau G., Pénot E., ig69. Répartition des cellules entre les tissus du rat adulte, préalablement soumis à une sous nutrition énergétique temporaire à deux stades de la croissance. $A n n$. Biol. anim. Bioch. Biophys, 9, 55-73.

Dirand G., Pénot E., ig6g. Évolution du nombre et de la taille des cellules dans les tissus de la rate amaigrie à la suite d'une carence énergétique. Croissance compensatrice. Ann. Biol. anim. Bioch. Biophys., 9, 575-587.

Kayser J., Neumane J., Lavollay J., ig66. Sur la longévité du rat Wistar nourti ad libitum avec un régime semi-synthétique. C. $R$. Acad. Sci., 262, 2063-2065.

Lister D., MCCANCE R. A., 1967. Severe undernutrition in growing and adult animals I7. The ultimate results of rehabilitations: Pigs. Br.J. Nutr., 21, 787-799.

McC $_{A y}$ C. M., CRowell M. F., MAYnaRd L. A., 1935. The effect of retarded growth upon the lenght of life span and upon the ultimate body size. J. Nutr., 10,63-79. 
McMeekan C. P., I940. Growth and development in the pig with special reference to carcass quality characteristics. J. Agric. Sci., 30, 387-436.

McMeEkan C. P, I94I. Growth and development in the pig with special reference to carcass quality characteristics. J. Agric. Sci., 31, I-49.

Siperstein D. M., I92I. The effects of acute and chronic inanition upon the development and structure of the testes in the albino rats. Anat. Rec., 20, 355-36r.

Widpowson E. M., MCCAnce R. A., r963. The effect of finite periods of undernutrition at different ages on the composition and subsequent development of the rat. Proc. R. Soc. B, 158, 329-342.

Widdowson E. M., Mavor W. O., McCANCE R. A., I964. The effect of undernutrition and rehabilitation on the development of the reproductive organs : rats. J. Endocr., 29, I I9-126. 